\title{
Application of Switched System to Train Dynamics Simulation
}

\author{
Lijuan Wan ${ }^{1, a}$, Santong Zhang ${ }^{1, b}$, Haifeng Wang ${ }^{1, c}$ \\ ${ }^{1}$ School of Electronic and Information Engineering, Beijing Jiaotong University, Beijing 100044,China \\ a13120279@bjtu.edu.cn, bstzhang@bjtu.edu.cn, chfwang@bjtu.edu.cn
}

Keywords: CTCS-3, Hybrid System, Switched System

\begin{abstract}
Switched System is an important method of analyzing the Hybrid System. CTCS-3 is a typical Hybrid System. To express the evolution of system states driven by events and time, Switched System is introduced into the analysis of CTCS-3. Meanwhile, the CTCS-3 has many operating modes. When the operating modes switch, large changes occur in the parameters of system, making it difficult to accurately describe the system changes with a single dynamic model, and Switched System can effectively solve this problem. Under these circumstances, Switched System is applied to the analysis of CTCS-3. In this paper, the scenario of the tracking between two trains is simulated. Simulation results show that Switched System has advantages on simplifying models, accurately describing the dynamic characteristics of a Hybrid System and adapting to the changes of the simulation environment.
\end{abstract}

\section{Introduction}

Hybrid system is a kind of complex system which both contains continuous dynamic characteristics, but also contains discrete dynamic characteristics, and two dynamics interact closely[1]. Switched system is an important branch of Hybrid system. Switched system comprises several subsystems and a switching law, the system switches among the subsystems according to the switching law. And each subsystem is the continuous dynamic part of the Switched system, while the switching law is the discrete part, the organic combination of them constitutes the entire behavior of the system.

As for China Train Control System (CTCS), time is not the only continuous variable in the system, velocity, acceleration, displacement also change continuously in the system. And the discrete states like operating mode, brake level, etc. changing with the external input, but also with the interaction of the continuous states. Thus a complex dynamic process is expressed.

This paper uses Switched system for modeling a track problem between two trains. According to the difference of operating modes, the system is divided into several subsystems. The paper analyses the behaviors of states either continuous or discrete under same or different modes to illustrate the feasibility of the method.

\section{Switched System}

Switched system is an important method to analyze Hybrid system[2], the basic idea is to add the discrete parts into a continuous system model[3]. In each subspace, the continuous states evolves with the law of subspace, when the evolution of the continuous states exceed the boundary of subspace, a system switch occurs, and system go into another subspace, then the continuous states will evolve with a new law.

The state equation of Hybrid system in subspace i can be expressed as[4]:

$$
H_{i}: x_{i, k}=f_{i, k}\left(x_{i, k}, v_{k-1}\right) \quad x_{i, k} \in \Omega_{i}, k \in \mathbb{N}
$$

$H_{i}$ denotes the discrete mode of subspace $\mathrm{i}, \mathrm{k}$ is discrete point of time, $f_{i, k}: \mathfrak{R}^{n_{x}} \times \mathfrak{R}^{n_{v}} \rightarrow \mathfrak{R}^{n_{x}}$ is a non-linear or linear function of the state $x_{i, k-1},\left\{v_{k-1}, k \in \mathbb{N}\right\}$ is an i.i.d process noise sequence, and $n_{x}, n_{v}$ denotes the dimension of the state vector and the process noise vectors. 
The measurement equation of system can be expressed as:

$$
y_{k}=h_{i, k}\left(x_{k}, \omega_{k}\right)
$$

$h_{i, k}: \mathfrak{R}^{n_{x}} \times \mathfrak{R}^{n_{\omega}} \rightarrow \mathfrak{R}^{n_{y}}$ is a non-linear or linear function, $\left\{\omega_{k}, k \in \mathbb{N}\right\}$ is an i.i.d observation noise sequence, And $n_{\omega}, n_{y}$ denotes the dimension of the observation noise vector and the observation vectors.

\section{Tracking Model}

The differential motion equation of the train is gave as:

$$
\frac{d v}{d t}=\frac{C}{(1+\gamma) \cdot m}=\frac{g \cdot c}{1000(1+\gamma)}=\xi \cdot c
$$

$C$ is the resultant force acting upon the train $(\mathrm{KN}) . c$ is the resultant force corresponding the unit gravity $(\mathrm{N} / \mathrm{KN}) . \mathrm{g}$ is the gravitational acceleration. $\gamma$ is the rotation quality factor, in general, $\gamma=0.06$. $\xi$ is the acceleration factor, whose value is determined by the rotation quality factor and the unit of acceleration.

For the single particle model of train, there are three forces mainly acting upon the train: tractive force, resistance, brake force. The unit resultant force c can be shown in Table 1.

Table 1 calculation of $\mathrm{c}$

\begin{tabular}{|c|c|c|}
\hline Operating mode & Unit resultant $(\mathrm{N} / \mathrm{KN})$ & Acceleration $(\mathrm{km} /(h \cdot s))$ \\
\hline traction & $c=f-w_{0}$ & \\
\hline cruise & $c=0$ & \multirow{2}{*}{$\mathrm{a}=\mathrm{c} / 30$} \\
\hline coast & $c=-w_{0}$ & \\
\hline Service Brake & $c=-b_{S B}-w_{0}$ & \\
\hline emergency brake & $c=-b_{E B}-w_{0}$ & \\
\hline
\end{tabular}

$f$ is the unit basic tractive force, $b_{S B}$ is the unit service brake force, $b_{E B}$ is the unit emergency brake force, $w_{0}$ is the unit resistance.

$w_{0}$ is usually described as:

$$
w_{0}=A+B v+C v^{2}
$$

$A, B, C$ are empirical constants. For the type of CRH2, which equipped with CTCS-3,the formula(4) can be expressed as:

$$
w_{0}=0.88+0.0074 v+0.000114 v^{2}
$$

Using the method of curve fitting can get the traction formula from figure 1:

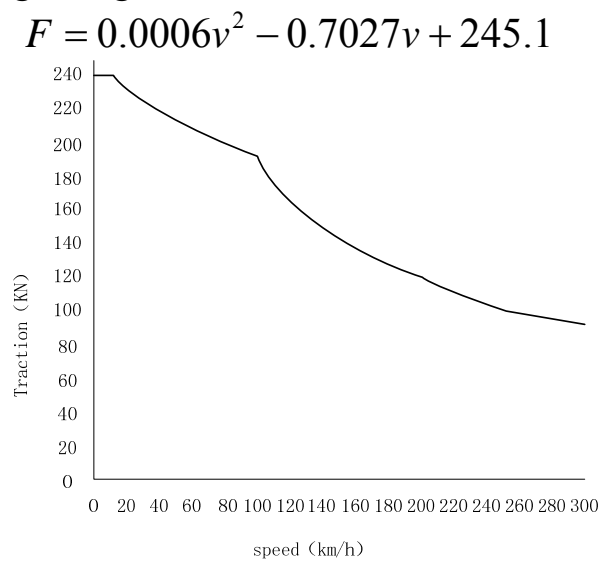

Fig.1 Traction characteristics curve

And the acceleration of service bake and emergency brake can get from figure 2 : 


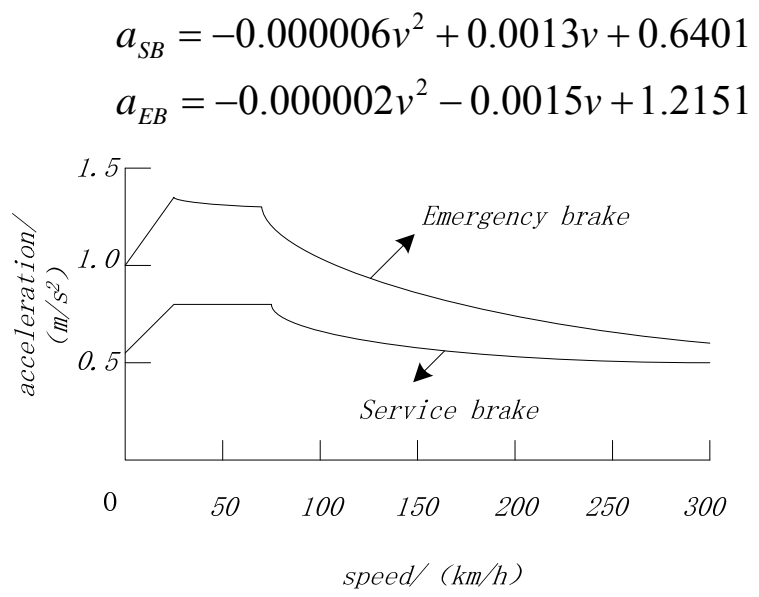

Fig.2 Braking characteristic curve

For CTCS-3, there are four speed limit curves with similar shape: Warning curve $\left(v_{w}\right)$, Permitted speed curve $\left(v_{p}\right)$, Service brake curve $\left(v_{S B I}\right)$, Emergency brake curve $\left(v_{E B I}\right)$. And a speed limit curve is comprised of CSM(Ceiling Speed Monitor) part and TSM(Target Speed Monitor) part, the figure 3 shows the emergency brake curve.

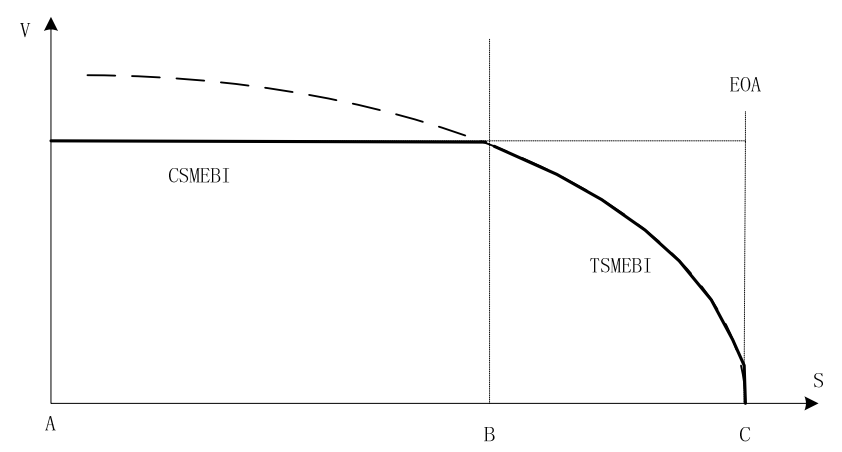

Fig.3 The emergency brake curve

And a limit speed $v_{E B I}$ can get from the emergency brake curve, so does the $v_{w}, v_{p}, v_{S B I}$.

$$
v_{E B I}=\min (C S M E B I, T S M E B I)
$$

According to the idea of Switched system, the system is divided into five subspaces, $H=\{1,2,3,4,5\}$ denotes the conditions: traction, cruise, coast, service brake and emergency brake. A state vector $x=\{s, v\}$ is defined to describe the continuous characteristics, $s$ represents the displacement of the train, $v=\dot{s}$ is the train's speed.

The state equation can be expressed as:

$$
x(k+1)=\left(\begin{array}{cc}
1 & T_{s} \\
0 & 1
\end{array}\right) x(k)+a \cdot\left(\begin{array}{c}
\frac{T_{s}^{2}}{2} \\
1
\end{array}\right)+\omega_{k}
$$

$T_{s}$ denotes sampling interval, $\omega_{k}$ is an i.i.d process noise sequence, $a$ denotes the acceleration, changes with the operating modes and speed.

The measurement equation can be expressed as:

$$
z(k)=\left(\begin{array}{ll}
1 & 0 \\
0 & 1
\end{array}\right) x(k)+v_{k}
$$

$v_{k}$ is an i.i.d observation noise sequence.

If the $v_{w}, v_{p}, v_{S B I}, v_{E B I}$ are given in current time, the switching law can be expressed as: 


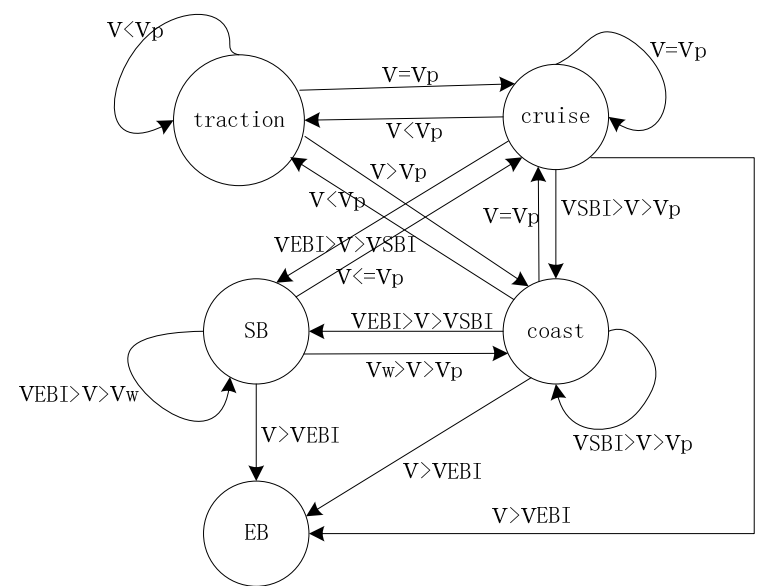

Fig.4 Switching law

\section{Result}

The basic parameters of CRH-2 are used in the simulation. Trains runs with the method of Quasi-moving block, means the tracking target of the latter train is the boundary of the block section where the front train is in.

Two trains runs at the same time, the starting interval is $3 \mathrm{~km}$ (while the length of block section is 2 $\mathrm{km}$ ). As is shown in figure 5, in the first 500 seconds, the interval between two trains expands gradually. After that, the interval becomes stabilized, about $7 \mathrm{~km}$ (larger than three block section).
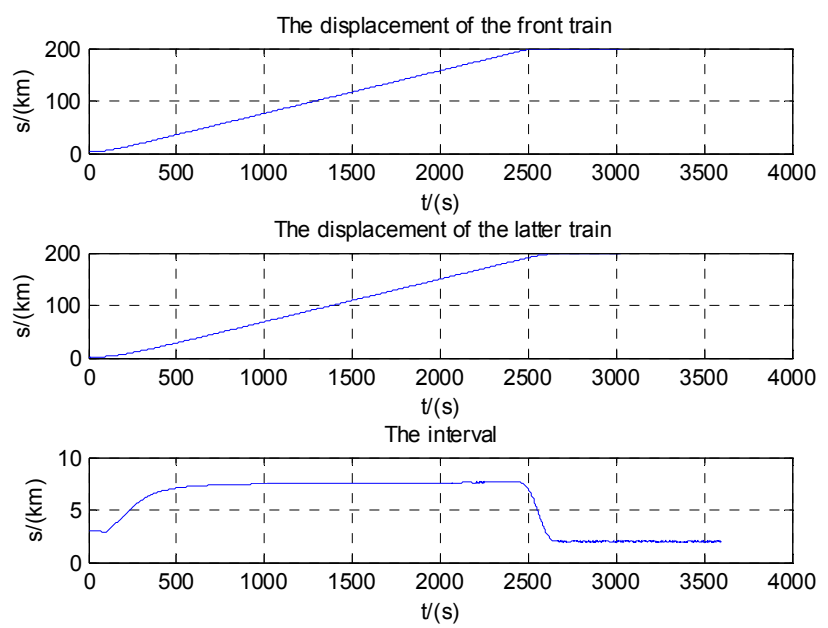

Fig.5 The displacement

The speeds of two trains are shown in figure 6. Obviously, trains accelerates in the first 500 seconds, then trains runs with the maximum speed, during this time, the interval between two trains is stabilized. 

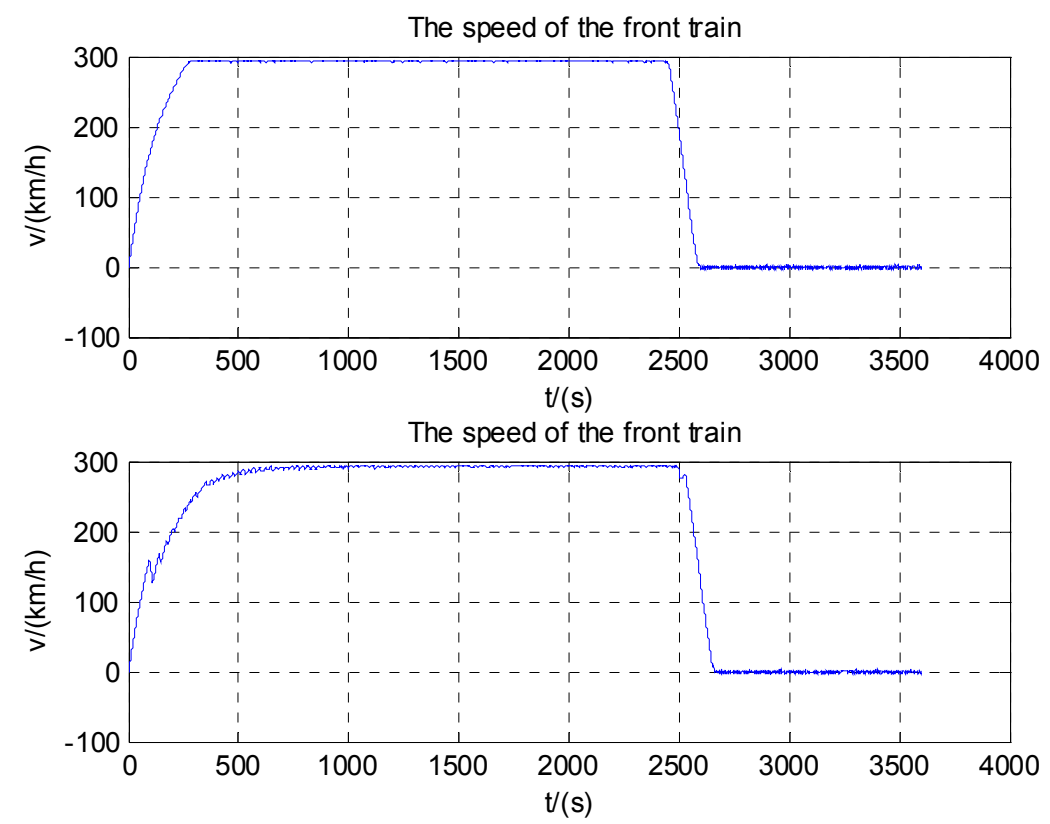

Fig.6 The speed

The acceleration of the front train under the traction mode is shown in figure 7 , the dynamic characteristics corresponds to the traction characteristics described in figure 1.

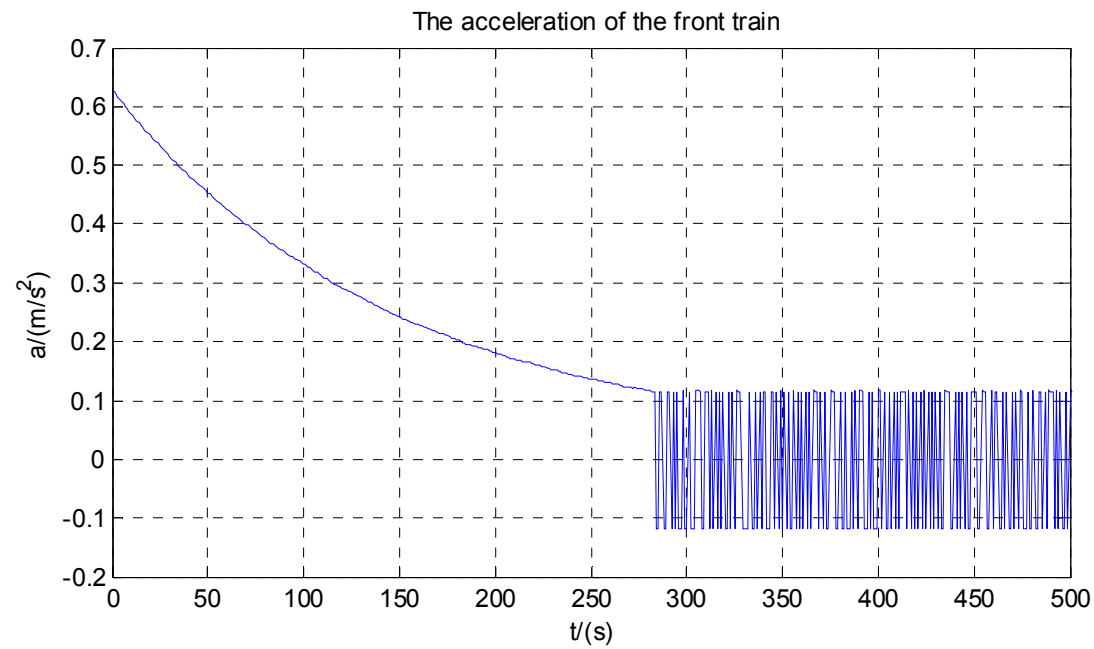

Fig.7 The acceleration of the front train

\section{Summary}

The paper applies the method of Switched system for the simulation of a tracking problem. Just design the state equations of subspaces and an additional switching law simplify the modeling process. The results shows that the method is effectively applied in the modeling of complex system.

\section{References}

[1]. Witsenhausen H S. A class of hybrid-state continuous-time dynamic systems[J]. IEEE Trans.on Automatic Control, 1966, 11(6): 665-683.

[2]. Cellier F E. Combined continuous/discrete system simulation by use of digital computer: techniques and tools[D]. Zurich, Switzer land: Swiss Federal Institute of Technology, 1979.

[3]. Antsaklis P J. Hybrid control systems: An introductory discussion to the special issue[J]. Automatic Control, IEEE Transactions on, 1998, 43(4): 457-460.

[4]. Alur R, Henzinger T A, Ho P H. Hybrid automata: an algorithmic approach to the 
specification and verification of hybrid systems[C]. Berlin, Germany: Springer-Verlag, 1993. 209-29.

[5]. Tafazoli S, Xuehong Sun. Hybrid system state tracking and fault detection using particle filters[J]. IEEE Transactions on Control Systems Technology, 2006, 14(6): 1078-1087. 